\title{
Deposição de filmes carbonosos em aço AISI D6 através da técnica de gaiola catódica
}

\author{
Deposition of carbonaceous films in AISI D6 \\ steel using the cathodic cage technique
}

\author{
Marcos Vinícius Soares Senna ${ }^{1,2}$, João Paulo Montalván Shica ${ }^{3}$, \\ Isaías Damasceno da Conceição ${ }^{2}$, Edgar Alves Araújo Júnior ${ }^{4}$, \\ Fernanda Roberta Marciano ${ }^{5}$, Rômulo Ribeiro Magalhães de Sousa ${ }^{1}$
}

\footnotetext{
${ }^{1}$ Laboratório de Plasma da UFPI - LAPLASMA-UFPI - PPGCM/CT/UFPI, Teresina, PI, Brasil.

${ }^{2}$ Programa de Pós-Graduação em Ciência e Engenharia dos Materiais, CT/UFPI, Teresina, PI, Brasil.

${ }^{3}$ Curso de Engenharia de Materiais, CT/UFPI, Teresina, PI, Brasil.

${ }^{4}$ Laboratório Interdisciplinar de Materiais Avançados (LIMAV/CT/UFPI), Teresina, PI, Brasil.

${ }^{5}$ Universidade Brasil, Instituto de Ciência e Tecnologia, São Paulo, SP, Brasil. e-mail:marcowsenna@hotmail.com,romulorms@gmail.com, isax12@ hotmail.com,eng.montalvan@gmail.com, edgar.aajr@gmail.com, frmarciano@pq.cnpq.br
}

\begin{abstract}
RESUMO
Neste trabalho, filmes finos de carbono tipo diamante DLC (Diamond-like carbon) foram depositados em substratos de aço ferramenta AISI D6 por meio da técnica de deposição a plasma com gaiola catódica de grafite, com o objetivo de avaliar a influência dos parâmetros de tratamento, tais como a duração do tratamento e a polarização das amostras. As amostras tratadas foram avaliadas em termos de morfologia e estrutura por Difração de Raios X (DRX), Espectroscopia Raman e Microscopia Eletrônica de Varredura (MEV). Os resultados dos difratogramas indicaram a presença das estruturas de ferrita, grafite e diamante para as amostras tratadas com alumina e a formação de grafite com estrutura hexagonal para amostras tratadas sem alumina. Os espectros Raman identificaram a banda D e G característicos de materiais grafíticos e os melhores resultados foram obtidos para as amostras que não utilizaram alumina. As micrografias dos filmes utilizando alumina indicaram estruturas na forma de minúsculas partículas, com contornos mais claros e definidos. Já para as amostras sem alumina, os filmes DLC apresentaram um aspecto mais uniforme e de coalescência dos grãos. As camadas das amostras tratadas com alumina apresentaram as maiores espessuras de camadas, principalmente para a amostra tratada durante 6 horas. Em relação aos tratamentos sem alumina, as amostras tratadas por 5 e 6 horas apresentaram em média a maior espessura de camada. Assim, foi possível afirmar que a alumina e, também, o tempo de tratamento influenciam de forma significativa nas características de microestrutura e espessura final do filme.
\end{abstract}

Palavras-chave: Deposição por Plasma, Filmes DLC e Gaiola Catódica.

\section{ABSTRACT}

In this work, thin films of diamond-like carbon DLC (Diamond-like carbon) were deposited on substrates of AISI D6 tool steel by means of the plasma deposition technique with cathodic cage of graphite, with the objective of evaluating the influence of the treatment parameters, such as the duration of the treatment and the polarization of the samples. The treated samples were evaluated in terms of morphology and structure by Xray Diffraction (XRD), Raman Spectroscopy and Scanning Electron Microscopy (SEM). The results of the diffractograms indicated the presence of the ferrite, graphite and diamond structures for the samples treated with alumina and the formation of graphite with hexagonal structure for treated samples without alumina. The Raman spectra identified the D and G bands characteristic of graphite materials and the best results were obtained for samples that did not use alumina. Micrographs of the films using alumina indicated structures in the form of tiny particles, with clearer and defined contours. As for the non-alumina samples, the DLC films presented a more uniform appearance and coalescence of the grains. The layers of the alumina treated samples had the highest layer thicknesses, especially for the treated sample for 6 hours. Regarding the treatments without alumina, the samples treated for 5 and 6 hours presented on average the highest layer thickness. Thus, 
it was possible to affirm that the alumina and, also, the time of treatment influence in a significant way the characteristics of microstructure and final thickness of the film.

Keywords: Deposition by Plasma, DLC Films and Cathode Cage.

\section{INTRODUÇÃO}

A maioria das ferramentas mecânicas metálicas são submetidas a elevados esforços mecânicos e ao desgaste $[1,2]$. Neste sentido, o aumento da demanda por maior produtividade e a redução do custo de fabricação requer melhorarias no desempenho e na longevidade das ferramentas de corte, punções, matrizes de forjamento, entre outros. Desta forma, faz-se necessário a melhoria das propriedades dos materiais de ferramentas existentes [3]. Neste trabalho foi utilizado o aço ferramenta AISI D6 comumente usados em punções e matrizes para operações de conformação a frio [4].

O aço ferramenta AISI D6 apresenta, relativamente, alto teor de carbono ( 2\%) e cromo ( 12,5\%), devido a transformações de fase, formam carbonetos de cromo que promovem a microdureza e a resistência ao desgaste. Apresentam características de elevada temperabilidade, alta resistência mecânica e ao desgaste, alto grau de indeformabilidade e boa tenacidade $[1,2,4]$.

A aplicação de filmes finos em componentes eletrônicos semicondutores e em revestimentos ópticos altera a superfície do substrato e melhora propriedades como dureza superficial, resistência ao desgaste e à corrosão e mantêm o núcleo tenaz. Os filmes finos à base de carbono têm ampla aplicação e suas características estruturais e propriedades são fortemente dependentes do método e taxa de deposição, do tipo de substrato, além da temperatura e pressão de aplicação [5, 6]. Além disto, a tensão residual é um dos principais motivos para a aplicação de revestimentos de DLC em aços, uma vez que influencia na estabilidade dos filmes nos substratos devido a incompatibilidade interfacial e diferença estrutural entre o filme e o substrato [5]. O carbono do tipo diamante consiste em uma variedade de materiais de carbono amorfo, como aC (carbono amorfo) e aC: H (carbono amorfo hidrogenado) que contêm frações significativas de ligações de carbono do tipo $\mathrm{sp}^{3}$, este confere propriedades físicas e mecânicas atraentes semelhantes ao diamante, como alta dureza, inércia química, alta transmitância óptica e suavidade da superfície [7].

Neste trabalho foi utilizado o processo de deposição de vapor químico por plasma, que consiste no confinamento de elétrons produzido por campos magnéticos e eventos de transferência de energia por indução [8]. Este é o método de deposição mais comum de filmes DLC, devido a um sistema de deposição simples, à baixa temperatura de trabalho e à baixa tensão residual nos filmes depositados $[9,10]$.

O objetivo deste trabalho foi depositar filmes finos de DLC em amostras de aço AISI D6, utilizando a técnica de deposição a plasma em gaiola catódica. Desta forma, foi avaliada a influência dos parâmetros de tratamento, tais como a duração do tratamento e o efeito da polarização das amostras com e sem a utilização de alumina durante o tratamento. Posteriormente, os filmes finos depositados foram avaliados em termos de estrutura e morfologia para evidenciar todas as fases formadas, além da espessura da camada e a regularidade do filme depositado.

\section{MATERIAIS E MÉTODOS}

As amostras utilizadas como substrato para a deposição dos filmes DLC são de aço ferramenta AISI D6. Foi utilizada uma gaiola catódica e uma tampa de grafite. Parte das amostras foram tratadas em potencial flutuante, no qual ficaram posicionadas sobre um disco de alumina e a outra parte foi posicionada diretamente sobre o porta-amostras onde ficaram aterradas.

\subsection{Preparação das amostras}

As amostras foram cortadas com as medidas $20 \times 10 \times 5 \mathrm{~cm}^{3}$ utilizando uma cortadeira metalográfica. Em seguida, cada amostra foi lixada e polida. Posteriormente, as amostras foram mergulhadas em solução de acetona e colocadas em equipamento de ultrassom para agitação durante 10 minutos, sendo secas em seguida com um secador comum.

As dimensões da gaiola catódica de grafite são: $40 \mathrm{~mm}$ de diâmetro, $35 \mathrm{~mm}$ de altura e $5 \mathrm{~mm}$ de espessura. As paredes possuem furos com $9 \mathrm{~mm}$, sendo a distância entre os centros dos mesmos de $15 \mathrm{~mm}$. Antes de ser utilizada na deposição, a gaiola e a tampa passaram por um leve lixamento para remoção de possíveis impurezas. O disco de alumina utilizado como base isolante possui $30 \mathrm{~mm}$ de diâmetro e $3 \mathrm{~mm}$ de espessura. 


\subsection{Parâmetros de tratamento}

A Tabela 1 apresenta os parâmetros de tratamentos utilizados neste trabalho. Assim, atmosfera, pressão e temperatura foram mantidas constantes, tendo como variáveis o tempo de tratamento e o uso ou não de alumina.

Tabela 1: Parâmetros de tratamento para a deposição com gaiola catódica com e sem alumina em aço AISI D6.

\begin{tabular}{c|c|c|c|c}
\hline Alumina & $\begin{array}{c}\text { Atmosfera } \\
\text { (sccm) }\end{array}$ & $\begin{array}{c}\text { Pressão } \\
\text { (Torr) }\end{array}$ & $\begin{array}{c}\text { Temperatura } \\
\left.\text { ( }{ }^{\circ} \mathbf{C}\right)\end{array}$ & $\begin{array}{c}\text { Tempo } \\
\text { (h) }\end{array}$ \\
\hline \multirow{4}{*}{$\operatorname{sim}$} & $4 \mathrm{Ar} / 16 \mathrm{H}_{2}$ & 1,5 & 400 & 4 \\
\cline { 2 - 5 } & $4 \mathrm{Ar} / 16 \mathrm{H}_{2}$ & 1,5 & 400 & 5 \\
\cline { 2 - 5 } & $4 \mathrm{Ar} / 16 \mathrm{H}_{2}$ & 1,5 & 400 & 6 \\
\hline \multirow{4}{*}{ não } & $4 \mathrm{Ar} / 16 \mathrm{H}_{2}$ & 1,5 & 400 & 4 \\
\cline { 2 - 5 } & $4 \mathrm{Ar} / 16 \mathrm{H}_{2}$ & 1,5 & 400 & 5 \\
\cline { 2 - 5 } & $4 \mathrm{Ar} / 16 \mathrm{H}_{2}$ & 1,5 & 400 & 6 \\
\hline
\end{tabular}

\subsection{Caracterizações das amostras}

A técnica de Difração de Raios X (DRX) foi realizada para identificar as fases presentes nos filmes finos depositados nas amostras. Foi utilizado um equipamento da marca PHILIPS modelo X'PERT MPT, com tubo de cobalto, tensão de operação de $40 \mathrm{kV}$ e corrente de $45 \mathrm{~mA}$, realizado no Laboratório de Caracterização de Materiais (LACAM-UFC).

A qualidade e as propriedades estruturais dos revestimentos de carbono foram avaliadas por Espectroscopia Raman em equipamento da marca BRUKER modelo SENTERRA, com feixe de radiação laser com comprimento de onda de $785 \mathrm{~nm}$. Foi realizado no Laboratório de Física dos Materiais (FISMAT-UFPI).

A morfologia da camada depositada nas amostras foi avaliada qualitativamente por Microscopia Eletrônica de Varredura, em equipamento da marca FEI COMPANY, modelo QUANTA FEG 250, realizado no Laboratório Interdisciplinar de Materiais Avançados (LIMAV-UFPI).

\section{RESULTADOS E DISCUSSÃO}

\subsection{Análise por Difração de Raios X (DRX)}

A Figura 1 apresenta os difratogramas das amostras tratadas com alumina durante 6, 5 e 4 horas e tratadas sem alumina por 6, 5 e 4 horas. Para as amostras tratadas com alumina, foi constatado uma predominância de picos em $2 \theta$ de aproximadamente $44,33^{\circ}$ referente ao carbono no plano (103) (carta JCPDS 26-1083) resultado semelhante foi encontrado em RAWAL et al. [11] e os picos $44,33^{\circ}, 64,6^{\circ}$ e $82,2^{\circ}$ se referem à ferrita $(\alpha-$ Fe) nos planos (200), (200) e (211), respectivamente (carta JCPDS 06-0696), resultado semelhante foi encontrado em ZHANG et al. [12].
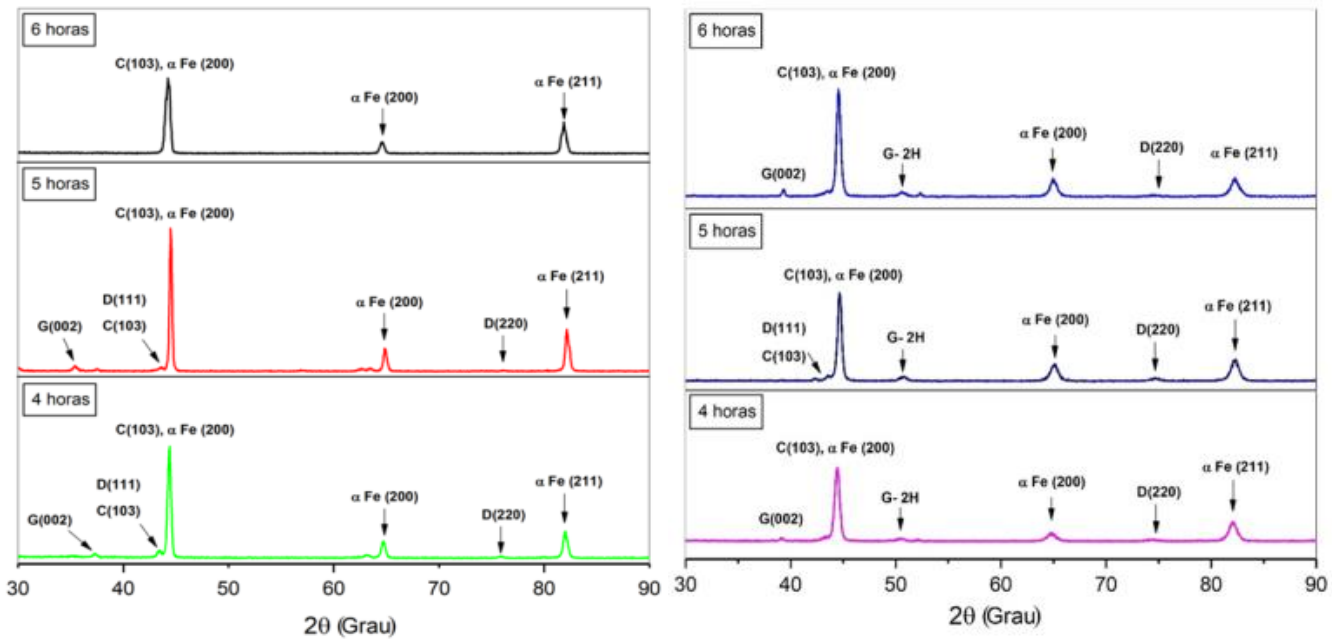

Figura 1: Difratogramas das amostras tratadas com alumina durante 6, 5 e 4 horas e as amostras tratadas sem alumina durante 6,5 e 4 horas. 
O pico em $35,2^{\circ}$ se refere à estrutura de grafite $(\mathrm{G})$ no plano (002) (carta JCPDS 74-2329) resultado semelhante foi encontrado por SOUZA et al. [13] evidenciando a estrutura hexagonal do carbono. Os respectivos picos em $2 \theta$ iguais a $43,72^{\circ}$ e $76,02^{\circ}$ se referem às estruturas de diamante (D) nos planos (111) (carta JCPDS 06-0675) resultado semelhante foi encontrado por FRANSEN et al. [14], onde atribui a este plano falhas de empilhamento que podem estar relacionados a uma taxa de crescimento rápido e D (220) (carta JCPDS 75-410) resultado semelhante foi encontrado em SHARDA et al. [15].

No entanto, na amostra com maior tempo de tratamento (6 horas), não foi identificado os picos característicos de grafite e diamante, o que indica que o tratamento realizado não favorece o surgimento dessas estruturas, além disto, apresenta uma superfície relativamente plana e com pouca rugosidade. Já as amostras que foram tratadas com alumina durante 4 e 5 horas apresentaram os melhores resultados, pois surgiram novos picos característicos de grafite e diamante com maior intensidade, indicando a presença dessas estruturas na película formada. JIN et al. [16] encontraram um pico característico de carbono diamante em $43,8^{\circ}$ na camada exterior da amostra tratada, correspondente ao plano (111) de cristal de diamante com estrutura cúbica de face centrada, o que implica a existência de ligação carbono $\mathrm{sp}^{3}$. Picos característicos da fase carbono tipo diamante (DLC) foram encontrados em $2 \theta$ igual a $76,02^{\circ}$, resultado semelhante foi encontrado por LIU et al. [5].

Para as amostras tratadas sem alumina, foram observados os mesmos picos apresentados nas amostras tratadas com alumina, no entanto, apresentaram menor intensidade para os picos de ferrita $(\alpha-\mathrm{Fe})$. Também foi possível observar o surgimento de um pico de grafite hexagonal $(\mathrm{G}-2 \mathrm{H})$ em aproximadamente $2 \theta$ igual a 50,76 (carta JCPDS 89-7213) resultado semelhante foi encontrado por REYES et al. [17] e também em PANG et al. [18] no qual identificaram o pico de grafite-2H com plano (102) em 50,36 ${ }^{\circ}$. Estas características podem contribuir na morfologia do filme, implicando em um filme menos regular e com maior rugosidade.

\subsection{Espectroscopia Raman}

A Figura 2 apresenta os espectros Raman avaliados na faixa de 1000 a $1800 \mathrm{~cm}^{-1}$ das amostras tratadas com e sem alumina. É possível observar as bandas D e G referentes aos materiais grafíticos que foram destacadas em faixas específicas no gráfico.
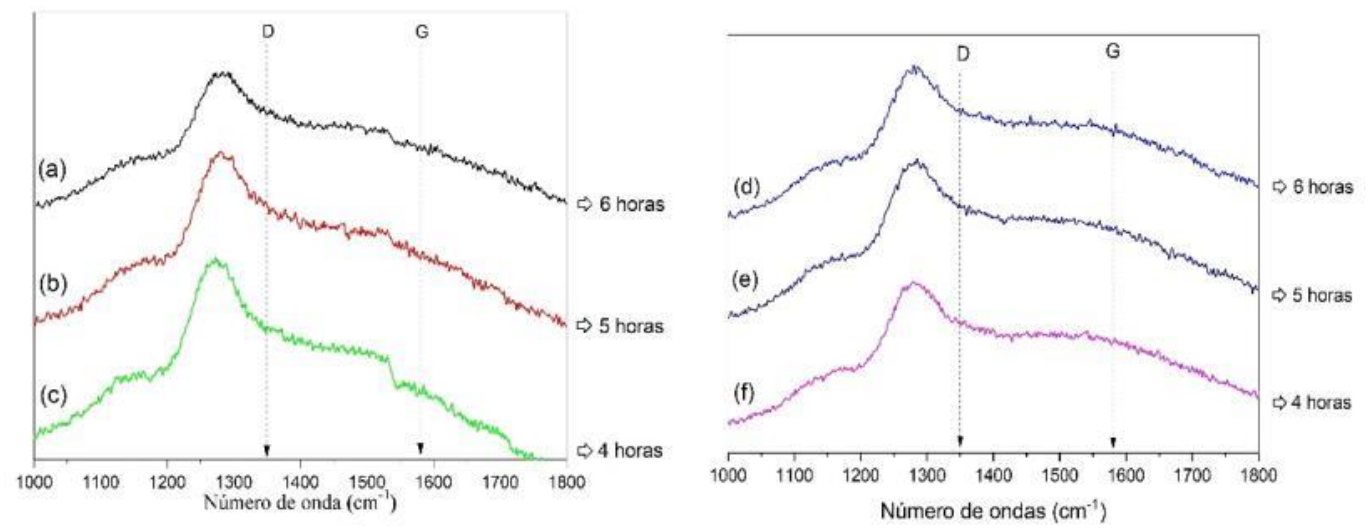

Figura 2: Espectroscopia Raman das amostras (a), (b) e (c) tratadas com alumina e (d), (e) e (f) tratadas sem alumina.

A banda D em torno de 1200 a $1450 \mathrm{~cm}^{-1}$ é ativada por desordem e ocorre apenas quando os sítios $\mathrm{sp}^{2}$ estão presentes em anéis aromáticos. Esta banda é atribuída ao modo de vibração das ligações de carbono (breathing mode), sendo originada de defeitos estruturais característicos de materiais carbonosos semelhante a grafita. A banda $\mathrm{G}$ em torno de 1550 a $1700 \mathrm{~cm}^{-1}$ corresponde a vibrações planares de átomos de carbono e está presente na maioria dos materiais semelhantes a grafita. Além disto, está relacionada ao modo de vibração de alongamento de todos os pares de sítios $\mathrm{sp}^{2}$ em anéis aromáticos e cadeias $\mathrm{C}=\mathrm{C}$ [19].

De forma complementar, foi realizada a análise da espectroscopia Raman obtendo a relação de intensidade $\mathrm{I}_{\mathrm{D}} / \mathrm{I}_{\mathrm{G}}$ através de ajustes nas curvas de cada espectro, utilizando curvas gaussianas e em seguida, foi aplicado a deconvolução gaussiana. Por fim, os valores de $\mathrm{I}_{\mathrm{D}}$ e $\mathrm{I}_{\mathrm{G}}$ apresentados na Tabela 2 foram obtidos através da integração das áreas correspondente a cada banda. Este método de cálculo de $\mathrm{I}_{\mathrm{D}}$ e $\mathrm{I}_{\mathrm{G}}$ foi baseado em FERRARI [20]. A razão de intensidade e a posição do pico G tem sido amplamente utilizada para estimativa qualitativa do conteúdo de $\mathrm{sp}^{3}$ em filmes DLC [19]. A fração $\mathrm{sp}^{3}$ é inversamente proporcional a relação de banda $\mathrm{I}_{\mathrm{D}} / \mathrm{I}_{\mathrm{G}}$. Desta forma, a estrutura é grafitizada quando essa razão aumenta [18]. 
Tabela 2: Dados obtidos da relação de intensidade $\mathrm{I}_{\mathrm{D}} / \mathrm{I}_{\mathrm{G}}$.

\begin{tabular}{|c|c|c|c|}
\hline Amostra & Alumina & Tempo (h) & $\mathbf{I}_{\mathbf{D}} / \mathbf{I}_{\mathbf{G}}$ \\
\hline $\mathrm{a}$ & \multirow{3}{*}{ Com } & 6 & 0,9903 \\
\hline $\mathrm{b}$ & & 5 & 0,8694 \\
\hline $\mathrm{c}$ & & 4 & 0,9120 \\
\hline $\mathrm{d}$ & \multirow{3}{*}{ Sem } & 6 & 0,4097 \\
\hline $\mathrm{e}$ & & 5 & 0,5891 \\
\hline $\mathrm{f}$ & & 4 & 0,5211 \\
\hline
\end{tabular}

Os melhores resultados foram obtidos para as amostras que não utilizaram alumina, principalmente a amostra (d) que apresentou razão de 0,4097, indicando mais ligações $\mathrm{sp}^{3}$ características de filmes DLC. Já as que utilizaram alumina apresentaram uma razão elevada, indicando maior presença de carbono grafite nos filmes depositados. Uma razão de 0,47 indica que a película fina de DLC depositada possui um conteúdo $\mathrm{sp}^{3}$ relativamente elevado e de alta qualidade [19]. Dentre as amostras tratadas utilizando alumina, pode-se observar que a amostra (b) é a que possui o menor valor da relação $\mathrm{I}_{\mathrm{D}} / \mathrm{I}_{\mathrm{G}}(0,8694)$, indicando que esta amostra possui a maior quantidade de $\mathrm{sp}^{3}$.

\subsection{Microscopia Eletrônica de Varredura (MEV)}

A Figura 3 apresentada as micrografias usando os elétrons secundários para as amostras tratadas com e sem alumina.

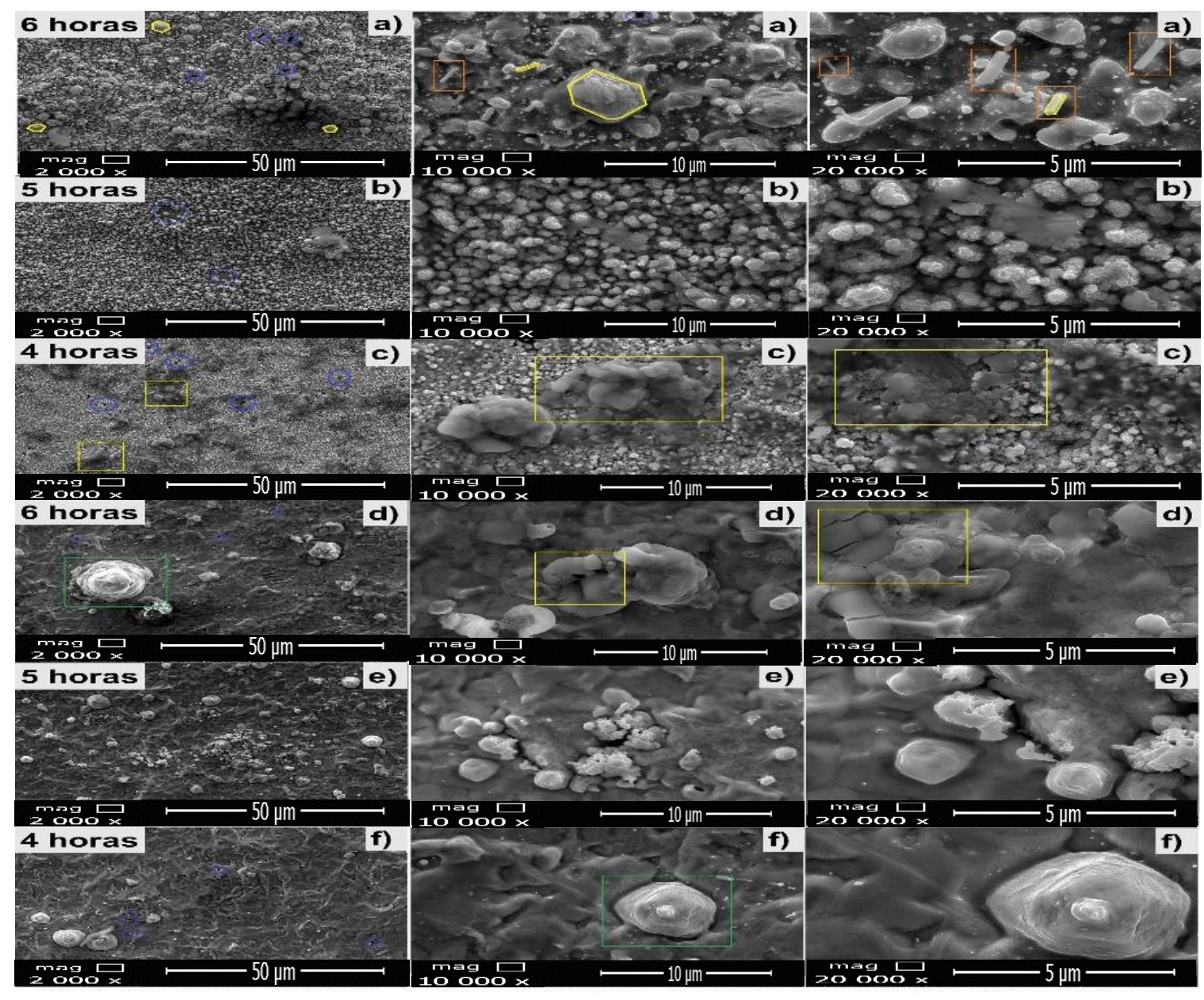

Figura 3: Micrografias MEV representativas das amostras tratadas por 6 (a), 5 (b) e 4 (c) horas com alumina e 6 (d), 5 (e) e 4 (f) horas sem alumina. 
A partir das micrografias foi possível observar uma diferença significativa na formação dos filmes utilizando alumina nas amostras do filme DLC, no qual está distribuído na forma de minúsculas partículas, com contornos mais claros e definidos, semelhante a carbonetos, além disso, apresentaram alguns espaços nas microestruturas. Verificou-se uma maior rugosidade das amostras tratadas com alumina pois possivelmente ocorreram menor difusão e coalescência dos grãos depositados na superfície dessas amostras, onde apresentaram aspecto heterogêneo. Já quando foi realizado o tratamento sem alumina, os filmes DLC apresentaram um aspecto mais uniforme e coalescência dos grãos e, portanto, com menor rugosidade, devido a maior difusão dos átomos nas amostras. Estudos realizados por SOUSA [21] indicaram que o não uso de alumina cria tensões diferentes nas amostras, tornando a deposição dos filmes irregulares.
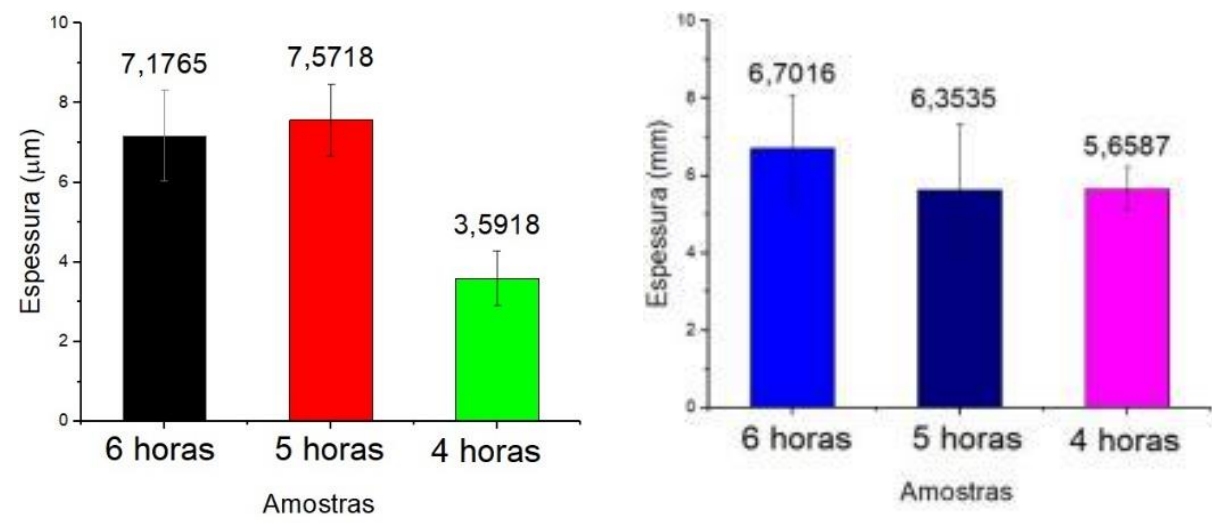

Figura 4: Média de espessura de camada das amostras tratadas durante 6, 5 e 4 horas com alumina e 6, 5 e 4 horas sem alumina.

Podemos afirmar que as amostras tratadas com alumina apresentaram as maiores espessuras de camadas, principalmente para a amostra com 5 e 6 horas de tratamento. Desta forma, com o maior tempo de tratamento apresentou melhor resultado. Já em relação às amostras tratadas sem alumina, a espessura de camada apresentou valores similares quando comparada com as tratadas utilizando alumina, no entanto, a amostra tratada por 6 horas apresentou em média a maior espessura de camada, a amostra tratada com o menor tempo apresentou a menor espessura de camada. Desta forma, foi possível identificar que a alumina e, também, o tempo de tratamento influencia de forma significativa na espessura final do filme depositado, de forma que os tratamentos utilizando alumina necessitam de mais tempo para que uma grande porção de filme seja depositado.

\section{CONCLUSÕES}

A partir dos resultados obtidos, conclui-se que o método utilizado para o tratamento das amostras apresentou resultados significativos. Assim, o DRX indicou a presença das estruturas de ferrita, grafite e diamante para as amostras tratadas com alumina e a formação de grafite com estrutura hexagonal para amostras tratadas sem alumina. O maior tempo de tratamento não favoreceu o surgimento dessas estruturas. Pelos resultados de Raman foi identificada a banda D que é ativada por desordem característica de materiais grafíticos e originase de defeitos estruturais e a banda G correspondente a vibrações planares de átomos de carbono. Os melhores resultados foram obtidos para as amostras que não utilizaram alumina, principalmente a amostra tratada por 6 horas que apresentou razão de 0,4097, indicando mais ligações $\mathrm{sp}^{3}$ características de filmes DLC. As micrografias dos filmes utilizando alumina indicaram estruturas na forma de minúsculas partículas, com contornos mais claros e definidos e alguns espaços nas microestruturas. Já para as amostras sem alumina, os filmes DLC apresentaram um aspecto mais uniforme e de coalescência dos grãos. Em relação às camadas, as amostras tratadas com alumina apresentaram as maiores espessuras de camadas. Desta forma, com o maior tempo de tratamento apresentou melhor resultado. Já em relação às amostras tratadas sem alumina, a amostra tratada por 6 horas apresentou em média a maior espessura de camada. Desta forma, foi possível identificar que a alumina e, também, o tempo de tratamento influencia de forma significativa nas características de microestrutura e espessura final do filme.

\section{AGRADECIMENTOS}

Os autores agradecem ao Programa de Pós-Graduação em Ciência e Engenharia dos Materiais (PPG- 
CEM/CT/UFPI), ao Laboratório de Plasma da Universidade Federal do Piauí (LabPlasma-UFPI), ao Laboratório de Caracterização de Materiais (LACAM/UFC), Laboratório de Física dos Materiais (FISMAT/UFPI) e ao Laboratório Interdisciplinar de Materiais Avançados (LIMAV/UFPI).

\section{BIBLIOGRAFIA}

[1] SILVA, A.L.C.E., MEI, P. R., Aços e ligas especiais, 3 ed., São Paulo, Edgard Blücher, 2008.

[2] VOURLiAS, G., CHALIAMPALIAS, D., ZORBA, T. T., et al., "A combined study of the oxidation mechanism and resistance of AISI D6 steel exposed at high temperature environments", Applied Surface Science, v. 257, n. 15, pp. 6687-6698, May 2011.

[3] DAS, D., RAY, K.K., "Structure-property correlation of sub-zero treated AISI D2 steel", Materials Science and Engineering: A, v. 541, pp. 45-60, Apr. 2012.

[4] BRESSAN, J. D., BATTISTON, G.A., ICIS, I., et al., "Wear on tool steel AISI M2, D6 and 52100 coated with $\mathrm{Al} 2 \mathrm{O} 3$ by the MOCVD process", Journal of materials processing technology, v. 179, n. 1-3, pp. 81-86, Oct. 2006.

[5] LIU, E., LI, L. BLANPAIN, B., CELIS, J. P., "Residual stresses of diamond and diamondlike carbon films", Journal of Applied Physics, v. 98, n. 7, pp. 1-5, Oct. 2005.

[6] USMAN, A., RAFIQUE, M. S., SHAUKAT, S. F., et al., "Impact of Argon gas on optical and electrical properties of Carbon thin films", Physica B: Condensed Matter, v. 503, pp. 157-161, Dec. 2016.

[7] COŞKUN, Ö. D., ZERRIN, T., "Optical, structural and bonding properties of diamond-like amorphous carbon films deposited by DC magnetron sputtering". Diamond and Related Materials, v. 56, pp. 29-35, Jun. 2015.

[8] LIEBERMAN, M. A., LICHTENBERG, A. J., "Principles of plasma discharges and materials processing", John Wiley \& Sons, v. 53, pp. 1689-1699, 2005.

[9] GRILL, A., "Diamond-like carbon: state of the art", Diamond and Related Materials, v. 8, N. 2-5, pp. 428-434, Mar. 1999.

[10] SAFAIE, P., ESHAGHI, A., BAKHSHI, S. R., "Structure and mechanical properties of oxygen doped diamond-like carbon thin films", Diamond and Related Materials, v. 70, pp. 91-97, Nov. 2016.

[11] RAWAL, I., PANWAR, S., TRIPATHI, R. K., et al., "Structural and nanomechanical properties of nanocrystalline carbon thin films for photodetection", Journal of Vacuum Science \& Technology A, v. 33, n. 3, pp. 031-0501, Feb. 2015.

[12] ZHANG, S., WU, W., XIAO, X., et al., "Preparation and characterization of spindle-like Fe3O4 mesoporous nanoparticles", Nanoscale research letters, v. 6, n. 1, pp. 89, Jan. 2011.

[13] SOUZA, E. E. S., SANTOS, A.F.T.O., GONZAGA, I.M.D., et al., "Ruthenium-tin oxides-coated graphite felt: Enhanced active area and improved efficiency for the electrochemical generation of hydrogen peroxide", Ceramics International, v. 41, pp. 10293-10297, Apr. 2015.

[14] FRANSEN, M.J., TE NIJENHUIS, J., VASTERINK, J.H.A., et al., "Analysis of the surface morphology of cvd-grown Diamond films with x-ray diffraction”, Adv. X-ray Anal, v. 46, pp. 185-191, Jan. 2003.

[15] SHARDA, T., RAHAMAN, M. M., NUKAYA, Y., et al., "Structural and optical properties of diamond and nano-diamond films grown by microwave plasma chemical vapor deposition", Diamond and Related Materials, v. 10, n. 3-7, pp. 561-567. Mac-Jul. 2001.

[16] JIN, X., ZHANG, Y., CHEN, L., et al., "Preparation and tribological behaviors of DLC/spinel composite film on 304 stainless steel formed by cathodic plasma electrolytic oxidation", Surface and Coatings Technology, v. 338, pp. 38-44, Mar. 2018.

[17] MARTÍNEZ-REYES, J., ARCEO, L.G., VAZQUEZ, L.R., et al., "Microstructural Characterization of Large Area $\mathrm{C}_{60}$ Films Obtained by Conventional Microwave Oven Irradiation", World Journal of Nano Science and Engineering, v. 2, pp. 213-218, Dec. 2012.

[18] PANG, H., WANG, X., ZHANG, G., et al., "Characterization of diamond-like carbon films by SEM, XRD and Raman spectroscopy", Applied Surface Science, v. 256, n. 21, pp. 6403-6407, Aug. 2010.

[19] SOHBATZADEH, F., SAFARI, R., ETAATI, G. R., et al., "Characterization of diamond-like carbon thin film synthesized by RF atmospheric pressure plasma Ar/CH4jet", Superlattices and Microstructures, v. 89, pp. 231-241, Jan. 2016. 
[20] FERRARI, A. C., "Raman spectroscopy of graphene and graphite: disorder, electron-phonon coupling, doping and nonadiabatic effects”, Solid state communications, v. 143, n. 1-2, pp. 47-57, Apr. 2007.

[21] SOUSA, R.R.M., "Nitretação em plasma com gaiola catódica: investigação do mecanismo e estudo comparativo com a nitretação em plasma de tensão continua”, Tese de D.Sc., PPGCEM/UFRN, Natal, RN, Brasil, 2007.

\section{ORCID}

Marcos Vinícius Soares Senna João Paulo Montalván Shica Isaías Damasceno da Conceição Edgar Alves Araújo Júnior Fernanda Roberta Marciano Rômulo Ribeiro Magalhães de Sousa https://orcid.org/0000-0001-9667-8235

https://orcid.org/0000-0002-1995-9380

https://orcid.org/0000-0002-6606-1442

https://orcid.org/0000-0001-7179-4010

https://orcid.org/0000-0001-7740-8766

https://orcid.org/0000-0003-2062-6505 